\title{
Legal area (Justice and Crime Coverage)
}

\section{AUTHOR}

Franziska Oehmer

\section{KEYWORDS}

judicial reporting, court reporting, representativity

\section{BRIEF DESCRIPTION}

It is often assumed that judicial reporting is biased in favor of criminal offences and violent crimes and at the expense of administrative, civil or labor court cases (e.g., Delitz, 1989; Eberle 1996; Machill, Beiler \& Hellmann, 2007). In order to be able to test this assumption, the variable "legal fields" is used to categorize the judicial trial or case reported in the media.

\section{FIELD OF APPLICATION/THEORETICAL FOUNDATION}

The legal field serves - among other variables - as an indicator of the representativeness of judicial reporting. Thus, the results of the content analysis are often compared with extra-media data on the distribution of trials in different fields of law (Strother, 2017).

\section{EXAMPLE STUDY}

Oehmer (work in progress)

\section{INFORMATION ON OEHMER (WORK IN PROGRESS)}

Authors: Franziska Oehmer

Research interest: The study focuses on three sets of questions concerning 1) the selection and representativeness of court reporting, 2) the information function of court reporting and 3) the presentation of court reporting.

Object of analysis: court coverage in Swiss newspapers (Tagesanzeiger, NZZ, Neue Luzerner Zeitung, Südostschweiz, Blick, Gratiszeitung, 20Minuten)

Time frame of analysis: January 2007 - December 2017

Codebook: available

\section{INFO ABOUT VARIABLES}

Variable name/definition: legal fields [Rechtsgebiete der berichteten Justizfälle]

Level of analysis: most covered court case in article

General coding instruction: The legal system is mostly characterized by a division of the legal fields into private law (or civil law) and public law. The following basic rule applies for the assignment to the legal areas: if only private individuals are involved, then it is private law (Code 10 ff.), if a public organization or a state is involved, then it usually refers to public law (Code $20 \mathrm{ff}$.). Unless otherwise stated, the following definitions are based on corresponding entries from the Gabler Wirtschaftslexikon. Springer, available at: https://wirtschaftslexikon.gabler.de/ (16.09.20)

\section{Variable name Values \& coding instructions}

Rechtsgebiet 10 Privatrecht: Privatrecht umfasst alle Rechtssätze, die die rechtlichen Bedes Prozes- ziehungen der einzelnen zueinander nach dem Grundsatz der Gleichordnung ses $\quad$ regeln. Der Staat oder ein anderer hoheitlicher Träger sind hier nicht beteiligt. Codierhinweis: Dieser Code wird gewählt, wenn keine Spezialform (Code 11f) vorliegt. 


\section{Variable name Values \& coding instructions}

Dazu zählen folgende Rechtsgebiete:

- Familienrecht

- Erbrecht

- Sachenrecht

11 Handelsrecht: Teilgebiet des Privatrechts. Handelsrecht ist das Sonderrecht des Kaufmanns. Die Vorschriften des Handelsrechts betreffen im Wesentlichen die Rechtsbeziehungen des Kaufmanns zu seinen Geschäftspartnern, die wettbewerbsrechtlichen und gesellschaftsrechtlichen Beziehungen zu anderen Unternehmern.

Dazu zählen folgende Rechtsgebiete:

- Kapitalmarktrecht,

- Wettbewerbsrecht,

- Versicherungsrecht

- Patentrecht

- Urheberrecht

- Markenrecht

12 Arbeitsrecht: zählt überwiegend zum Privatrecht. Gesamtheit aller Rechtsregeln, die sich mit der unselbstständigen, abhängigen Arbeit befassen, d.h. der Arbeit, die von Personen geleistet wird, die in einem Betrieb eingegliedert fremdbestimmte Arbeit leisten und dabei an Weisungen hinsichtlich Art, Ausführung, Ort und Zeit der Arbeit gebunden sind.

\section{Mietrecht}

20 Öffentliches Recht: regelt, im Gegensatz zum Privatrecht, die Beziehungen des Einzelnen zum Staat und den Körperschaften des öffentlichen Rechts sowie der Träger öffentlicher Gewalt zueinander. Im öffentlichen Recht ist der Einzelne (anders als im Privatrecht) dem Staat untergeordnet. Der Staat oder ein Träger hoheitlicher Gewalt tritt mit Hoheitsgewalt auf (Forstmoser/Vogt 2012, S.118)

Codierhinweis: Dieser Code wird gewählt, wenn keine Spezialform (Code 21f) vorliegt.

21 Verwaltungsrecht: Mit Verwaltungstätigkeit ist die Tätigkeit der öffentlichen Verwaltung gemeint. Die „öffentliche Verwaltung“ wird von den Einrichtungen der unmittelbaren und mittelbaren Staatsverwaltung (Bund, Ländern, Gemeinden, Gemeindeverbänden und sonstigen Körperschaften, Anstalten und Stiftungen des öffentlichen Rechts) gebildet (institutioneller Begriff). Quelle: Bader, Ronellenfitsch, 2016, § 1 Rn. 8-10.1).

Dazu zählen u.a. folgende Rechstgebiete:

- Verwaltungsgerichtsbarkeit

- Bauplanung, Naturschutz

- Ausländer, Staatsbürgerrecht

- Beamten/Soldatenrecht

- Schul/Hochschulrecht

- Verkehrs/Wegerecht

- Leistungs/Sozialrecht 
- Rundfunkrecht

- Gewerbe/Lebensmittel

- Waffenrecht

- Kommunalrecht

- Veranstaltungs-/Demonstrationsrecht

- Wohnungsrecht

22 Verfassungsrecht: Rechtliche Grundordnung eines Staates, Gesamtheit der geschriebenen und ungeschriebenen Rechtssätze über die Bildung, den Aufgabenkreis und die Organisation der obersten Staatsorgane, das Verhältnis der einzelnen Staatsorgane zueinander, die staatlichen Aufgaben, den staatsrechtlichen Aufbau des Staates und die Rechte des Bürgers gegen den Staat (Grundrechte) (Quelle: http://wirtschaftslexikon.gabler.de/Archiv/4350/oeffentlichesrecht-v5.html)

23 Steuerrecht: Gesamtheit der Rechtsnormen unserer Rechtsordnung, die sich - im weitesten Sinn - auf Steuern beziehen. Diese schaffen und regeln die Rechtsbeziehungen (Rechte und Pflichten) zwischen den Trägern der Steuerhoheit und den ihnen unterworfenen natürlichen und juristischen Personen.

24 Sozialrecht: Teilgebiet des öffentlichen Verwaltungsrechts. Das Sozialrecht soll zur Verwirklichung sozialer Gerechtigkeit und sozialer Sicherheit dienen. Dazu zählen folgende Rechtsgebiete:

- Sozialgerichtsbarkeit

- Unfallversicherung

- Rentenversicherung

- Krankenversicherung

- Kriegsopferversorgung

- Arbeitslosenversicherung

- Kassenarztrecht

\section{Internationales Recht}

26 Strafrecht (hier auch Jugendstrafrecht): Inbegriff der Rechtsnormen, in denen die Voraussetzungen für die Straftat und ihre Rechtsfolgen festgelegt sind; umfasst i.w.S. auch das Strafverfahrensrecht, das der Durchsetzung des staatlichen Strafanspruchs dient. Strafrecht ist Teil des öffentlichen Rechts, was nach allen gängigen Differenzierungstheorien (Subordinationstheorie, Interessentheorie, neuere Subjektstheorie) deutlich nachweisbar ist.

\section{Codierhinweis:}

a) Beim Strafrecht sind aufgrund seiner zu erwartenden Sonderstellung in der Berichterstattung möglichst detaillierte Codes zu vergeben.

b) Werden im Rahmen einer Verhandlung mehrere Delikte verhandelt, so wird das Delikt codiert, dem in der Berichterstattung der meiste Raum beigemessen wird. Werden sämtliche Delikte im gleichen Ausmass behandelt, so wird das Erstgenannte codiert.

$\begin{array}{ll}27 & \text { Tötung } \\ 28 & \text { Körperverletzung } \\ 29 & \text { Raub } \\ 30 & \text { Sexualdelikte } \\ 31 & \text { Eigentumsdelikte }\end{array}$


Variable name Values \& coding instructions

\begin{tabular}{ll}
\hline 32 & Gemeingefährliche Delikte \\
33 & Rauschgiftdelikte \\
34 & Delikte gegen die öffentliche Ordnung \\
35 & Beleidigung \\
36 & Amtsdelikte \\
37 & Ordnungswidrigkeit \\
& \\
$99)$ & Sonstiges
\end{tabular}

Intercoder reliability: Holsti .81; Krippendorff's

Alpha: .68 (2 Coder)

\section{REFERENCES}

Delitz, J. (1989). Tagespresse und Justiz. Gerichtsberichterstattung als Vermittlung institutioneller Wirklichkeit. Hamburg. [Daily press and justice. Court reporting as a mediator of institutional reality.]

Eberle, R. G. (1996). Verwaltungsgerichte in der Medienberichterstattung am Beispiel von Tageszeitungen in Hessen. Zeitschrift für Rechtssoziologie, 17(2), S. 300-309. [Administrative courts in media coverage using the example of daily newspapers in the federal state of Hesse.]

Bader, J. \& Ronellenfitsch, M. (2016). Verwaltungsverfahrensgesetz: VwVfG. Beck. [Administrative Procedure Act]

Forstmoser. P.\& Vogt, H-U. (2012). Einführung in das Recht. 5. Vollständig überarbeitete Auflage. Stämpfli. [Introduction to law.]

Machill, M., Beiler, M. \& Hellmann, I. (2007). The selection process in local court reporting. Journalism Practice, 1(1), S. 62-81.

Oehmer, Franziska. Die dritte Gewalt in den Medien. Eine repräsentative quantitative Inhaltsanalyse der Gerichtsberichterstattung Schweizer Medien (work in progress). [Justice in the media. A representative quantitative content analysis of court reporting in the Swiss media].

Strother, L. (2017). How expected political and legal impact drive media coverage of Supreme Court cases, Political Communication, 34(4), S. 571-589.Text 